\title{
An Efficient Hybrid Parallel Prefix Adders for Reverse Converters using QCA Technology
}

\author{
N. Chandini \\ M.Tech student Scholar \\ Dept.of ECE \\ AITAM
}

\author{
B. Chinna Rao \\ Associate Professor \\ Dept.of ECE \\ AITAM
}

\author{
A. Jaya Laxmi \\ Assistant Professor \\ Dept.of ECE \\ AITAM
}

\begin{abstract}
In many building blocks of microprocessors and digital signal processing chips, adders are frequently available in their critical paths. Adders can also be used for subtraction, multiplication and division. One of the important basic arithmetic operations is addition. There are several structures like Ripple Carry Adder (RCA), Carry Look Ahead Adder (CLA) to perform the addition. Parallel prefix adders speed up the addition operation when compared to the other structures. Generally these adders provide less power consumption, but these consume more power when these are used in reverse converters. To reduce this high power consumption, hybrid parallel prefix adders can be used. In this paper, two structures namely, Hybrid Regular Parallel Prefix XOR/OR (HRPX) Adder and Hybrid Modular Parallel Prefix Excess-one (HMPE) Adder are discussed which uses modulo addition. Further these two adders are implemented using the Quantum dot cellular automata (QCA) technology, which reduces the delay. This entire work is done in Xilinx 13.2 tool ISE simulator.
\end{abstract}

\section{Keywords}

Addition, Parallel prefix adders, Black cell, Gray cell, Quantum dot cellular automata, Power, Delay.

\section{INTRODUCTION}

The most fundamental and used arithmetic operation is the addition in Digital signal processing (DSP), microprocessors and data processing applications etc. So in VLSI, adders are the main building blocks. There are many structures for performing the addition operation. The hybrid adders are also retrieved from the basic designs. Certain adders like parallel prefix and conditional sum adders are important as they can easily fit in addition with special modulo sets. For the present technology the fastest and high performance obtained adders are the parallel prefix adders.

Since the complexity of Residue number system (RNS) is more, it cannot be directly implemented as same as conventional arithmetic. Therefore, it is realized using look up tables (LUT) and some standard arithmetic. This system is used in battery based devices as it consumes less power. It consists of three major blocks named as forward converter, arithmetic unit and reverse converter. The normal representation is converted to residue representation by the forward converter. The standard operations are present in the arithmetic block and the operations can be done on the moduli choice. Residue addition is accomplished by adding individual corresponding digits, relative to the modulus for their position. That is, a carry out from one position is not propagated into the next digit position. The process of converting reverse notation to normal representation is done in reverse converter.
Applications of RNS are digital signal processing, multiply and accumulate unit (MAC) and error detection and correction units, cryptography etc. RNS speeds up the complex number arithmetic and it is advantageous with respect to the arithmetic operations of addition and multiplication because of parallelism. In RNS reverse converter is the fundamental part as it consists of complex structures.

The rest of this paper is organized as follows. Section-II presents the structure of parallel prefix adder and its mathematical equations. Section-III explains the implementation of reverse converter structures i.e. HRPX and HMPE with prefix adders. The methodology of the proposed technique QCA with conventional reverse converters is presented in Section-IV. Results of total power and comparison table are discussed in Section-V. Section-VI is the conclusion of the work."

\section{OVERVIEW OF PARALLEL PREFIX ADDERS}

Parallel prefix adders are used in order to compute the carries in advance without delay and complexity and for wider bit lengths. Its name itself indicates that the addition is done in parallel fashion. In the three stages, the parallel prefix operation is done, which are pre calculating stage, calculation of carries and post processing stage. These adders are belongs to the family of carry look ahead adders and are of more speed. These adders support wide word lengths and uses tree network. There are different varieties of prefix adders and these adders differ in carry generation unit.

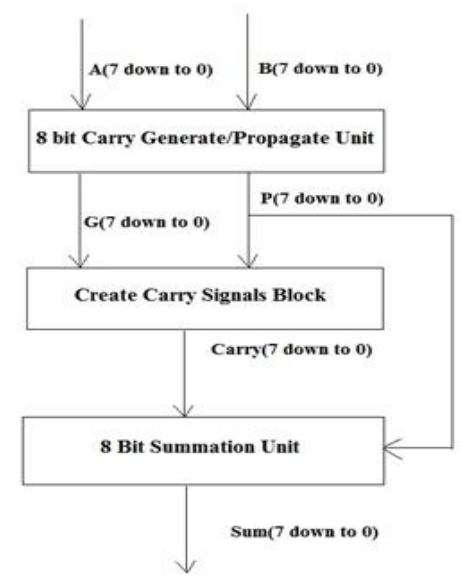

Fig 1: Block diagram of parallel prefix adder

The operation of the parallel prefix adder is explained in the following three steps.

Step 1: Pre calculation stage 
In this stage, the inputs are given and then propagate and generate unit calculates the carry generate and propagate based on the below formulae.

$$
\begin{aligned}
P_{i} & =A_{i} \text { xorB }_{i} \\
G_{i} & =A_{i} a B_{B}
\end{aligned}
$$

Step 2: Prefix calculation stage

Carry signals are generated by using different prefix graphs. These calculated values are forwarded to summation stage after calculation of these carry prefixes. The group generate/propagate signals are computed by using the below equations. The black cells and gray cells are included in this stage for easy computation of carry prefixes. The black cell (BC) generates ordered pair and the gray cells (GC) generate only left signals.

$$
\begin{gathered}
P=P_{i} \text { and } P_{i} \text { prev } \\
G=\left(P_{i} \text { and } G_{i \text { prev }}\right) \text { or } G_{i}
\end{gathered}
$$
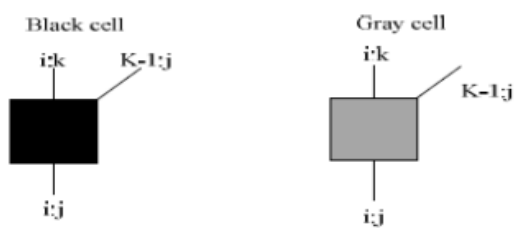

Fig 2: Representation of Black cells and gray cells

The operation is done in parallel fashion by decomposing into smaller pieces. The combined operator used in this stage consists of two AND gates and the OR gate. Each vertical stage gives out the respective propagate and generate values.

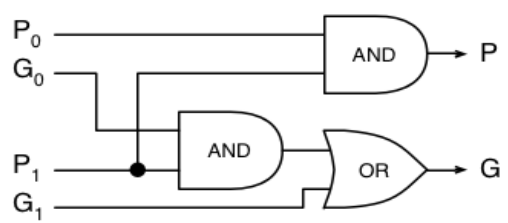

Fig 3: Block diagram of combined operator $\mathbf{P} \& \mathbf{G}$

Step 3: Summation stage

The calculated values of carries are forwarded to the post processing stage. The final sum values are calculated in this stage. A dot operator (.) is used in the simplification of generate and propagate terms.

$$
\begin{gathered}
S_{i}=P_{i} \operatorname{xor}_{i-1} \\
C_{i}=G_{i}
\end{gathered}
$$

Out of these three steps, 1 and 3 steps are fast as they involved with simple operations to each bit position. The step2 involves propagation of carries and the whole performance depends on this step. There are different kinds of prefix structures. They are:

- J.Sklansky - conditional adder.

- $\quad$ Ladner- Fisher adder.

- Kogge- Stone adder.

- $\quad$ Brent- Kung adder.

- Han Carlson adder.
Out of these adders, $\mathrm{KS}$ and BK adders are used in this work and are discussed below.

\subsection{Kogge Stone (KS) Adder}

The 8-bit KS adder is designed. By using this technique, adder is implemented with regular layout and a controlled fan-out. This adder is very useful for high speed applications. The delay and computational nodes of this adder are given by $\log _{2} n$ and [(n) $(\log 2 n)-n+1]$ respectively, where ' $n$ ' is the number of input bits. If a recursive doubling algorithm is introduced in the graph, there will be a problem with fan-out. To limit this fanout, idempotency property is used at the cost of increased number of wires at each stage. This is the fastest adder as it operates totally on generates and propagate blocks but consumes more area among all the parallel prefix adders.

Another advantage is that it uses fewer logic levels. This adder reduces the critical path when it is used in high performance 32bit, 64-bit and 128 bit etc.

\subsection{Brent Kung (BK) Adder}

The operational speed is reduced for BK adder because it uses large number of levels. It computes the prefixes for 2 bit groups. Based on these 2 bit groups, 4-bit, 8-bit and so on can be obtained The complex parallel prefix structure is Brent Kung (BK) adder as it brings about two tree structures. One is for carry store and other is for carry redistribution. Top view for the 8 bit BK adder is shown below. The delay and computational nodes for this adder is given by [(2) $\left.\left(\log _{2} n\right)-2\right]$ and $\left[2(n)-2-\log _{2} n\right]$ respectively. Here the number of BC's and GC's used are less compared to KS adder. So, the area required is less. The number of propagate and generate cells used are also less.

\section{HYBRID PARALLEL PREFIX ADDERS}

The usage of parallel prefix adders in reverse converter design has the problem of higher power dissipation. To avoid this problem, hybrid parallel prefix structures are preferred.

\subsection{HMPE Structure using KS adder}

In this paper, HMPE with KS adder is designed and it achieves fast operation, as it is computed in parallel fashion. This prefix adder is suitable for achieving a tradeoff between speed and cost by selecting the right adders. Based on the control signal generated by the prefix structure, the result from this structure is incremented. The excess- 1 unit is advantageous as it contains less number of logic gates when compared to the n-bit full adder structure. The HMPE is more flexible for any prefix structure. The performance parameters like power, delay and area are adjusted by selecting the required prefix adder. The top view and RTL schematic of HMPE structure implemented using KS adder are shown in Fig 4 and Fig 5 respectively.

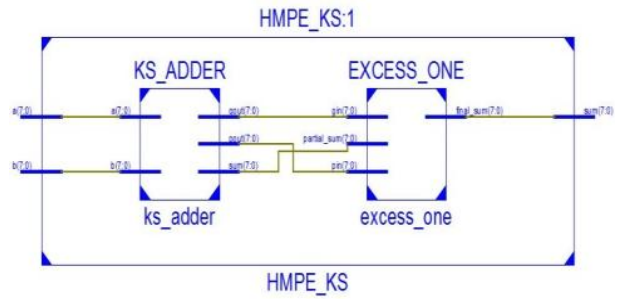

Fig 4: Top view of HMPE Structure using KS adder 


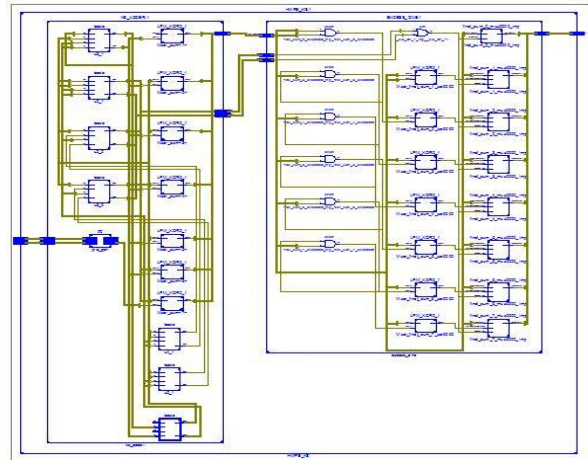

Fig 5: RTL schematic of HMPE Structure using KS adder

Generally, the carry-in depends on the carry-out in End Around Carry (EAC) adder. This means that the output carry is feedback to carry in through some logic, which is used in modulo additions. Both carry in and carry out generation processing conditions have to be met to obtain fast end around carry. With fast processing, the parallel prefix adder fulfills all these requirements. For modulo addition,

$$
\begin{array}{cl}
A+B=A+B+1 & \text { if } A+B \geq 2^{n} \\
A+B & \text { otherwise }
\end{array}
$$

The carry out obtained from this addition determines whether the carry out is normal or incremented carry out is added to sum. This results in double zero representation. For single zero representation, the condition $A+B \geq 2^{n}-1$ is fulfilled if either $A+B \geq 2^{n}$ or $A+B=2^{n}-1$ which corresponds to propagate signal of prefix adder. By using prefix adder, modulo addition with single zero representation can be easily implemented.

\subsection{HMPE Structure using BK adder}

The double zero representation can be resolved by the hybrid modular parallel prefix excess-1 structure. It contains two units namely regular prefix adder and the excess- 1 unit. The first the two operands are added and the result is incremented based on the control signals to produce a single zero representation. These structures can be implemented with the desired prefix adders. The top view and RTL schematic of HMPE structure implemented using BK adder are shown in Fig 6 and Fig 7 respectively.

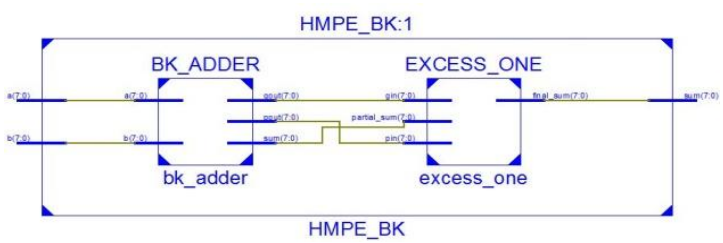

Fig 6: Top view of HMPE Structure using BK adder

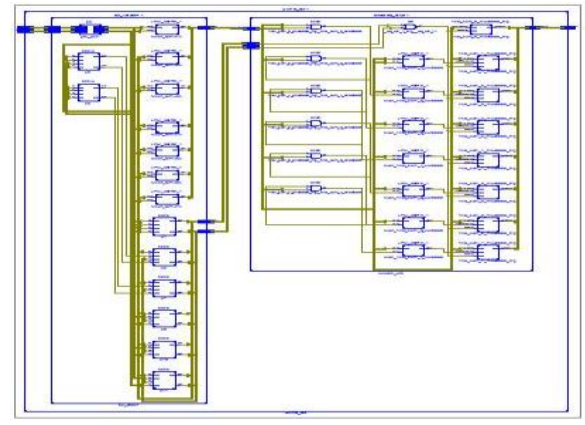

Fig 7: RTL schematic of HMPE Structure using BK adder

\subsection{HRPX Structure using BK adder}

A desired and regular parallel prefix adder is chosen for the first part of the addition, for which the corresponding bits are fully variable. A simplified logic with a RCA is used in which the full adders are replaced with XOR/OR gates for the operation of second part. The HRPX adder performs the $(4 n+1)$ addition, where $n$ value is 4 . The carry propagate adder with end around carry (EAC) with a moduli $2 n-1$ adder produces double zero representation. Single zero representation is sufficient in reverse converters. So, a detector is needed which increases the delay for this representation. The top view and RTL schematic of HMPE structure implemented using BK adder are shown in Fig 6 and Fig 7 respectively.

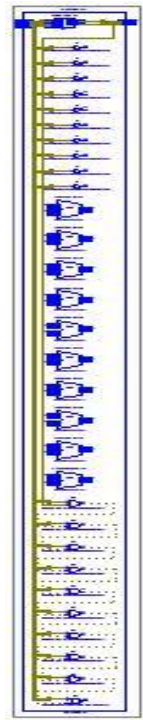

Fig 8: RTL schematic of HRPX Structure using BK adder

The first operand contains the upper bits of $2 \mathrm{n}$ of 1's. Addition of first $2 n+1$ bits is done by PP adder with $2 n+1$ bits and the next $2 n+1$ bits contains one's performs the ripple carry addition with carry of last $2 n+1$ bits. So, the speed of operation can be improved by pairing of RCA with parallel prefix adder. The number of gates is reduced if the full adder in RCA design is replaced with XNOR/OR gates. The BK adder is preferred compared to other adders, as it has minimum fan out and higher speed of operation. Therefore, the large size parallel prefix adder can be avoided. Due to the recursive effect of generating and propagating signals at each prefix level, this structure consumes high power and area.

\section{PROPOSED METHOD}

As today transistor size goes on decreasing, there is no scope to reduce its size further because of the parameters like tunneling effect, sub-threshold leakage, interconnect delay. To avoid this problem, a new technology has been emerged which is, Quantum dot cellular automata (QCA), a nanotechnology methodology. This decreases the speed with dense structures. The hybrid reverse converters discussed above are implemented with this technology in this work

This technology is based on field coupled computing. QCA cell is the basic component of QCA Logic. Due to the mutual interactions of either electrostatic or magnetic fields, there is a change in the state of a cell. Four quantum dots are present in each QCA cell, in which only two mobile electrons can be trapped that can tunnel between the dots. Two electrons are placed in diagonal position due to the repulsion between the electrons. In CMOS, binary values are represented in voltage 
or current levels. Just like that, electron position represents the binary values in this technology. The two basic logic devices of QCA are Majority gate and Inverter.

\subsection{Implementation of Hybrid Structures using QCA technology}

Using QCA technology, any parallel prefix adder can be implemented. Majority gate contains 5 QCA cells. Out of these five cells, 3 are input cells, one intermediate cell and one output cell. The operation of this majority gate is described by below equation.

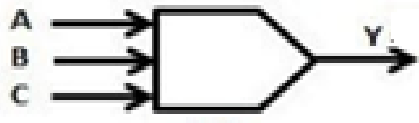

Fig 9: Representation of majority gate

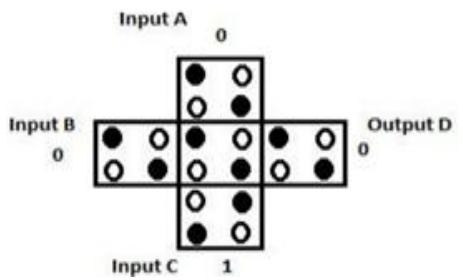

Fig 10: Schematic of Majority gate.

$$
M(a, b, c)=a \cdot b+a \cdot c+b \cdot c
$$

The majority gate output is calculated by using the above formula. There will be 8 combinations for three inputs. The above structures HRPX BK, HMPE BK and HMPE KS are implemented by using QCA technology. The entire operation is same as before. The only that make difference is the prefix adders are implemented using majority gates rather than CMOS logic gates. The implementation of BK and KS prefix adders with majority gates are shown in Fig.11 and Fig.12 respectively.

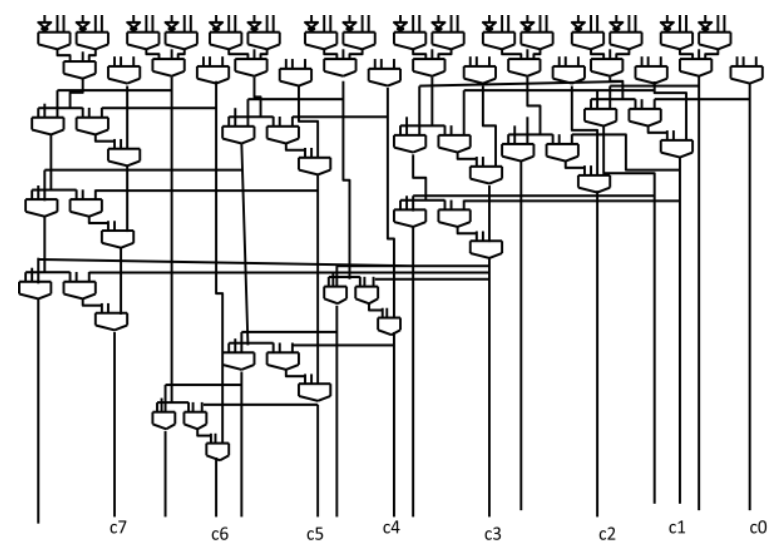

Fig 11: Brent Kung adder with majority gates

The implementation of $\mathrm{BK}$ and $\mathrm{KS}$ structures are done as follows. As the parallel prefix adders are implemented in of 3 stages, the first stage consists of propagate and generate units. The second stage consists of carry calculation of which it consists of 12 levels of majority gates. After that the final sum values are calculated by using these carries.

Similarly for KS adder, 3 stages are present. First stage consists of propagate and generate blocks. Second stage consists of majority gates of 6 levels of carry calculation and the obtained carry is forwarded to sum block to obtain modulo addition. The BK and KS adders are implemented in the HRPX and HMPE structures using this technology. In HMPE structure, modified excess one unit is also designed along with prefix adder. The modified excess unit consists of xor gates and the AND gates.

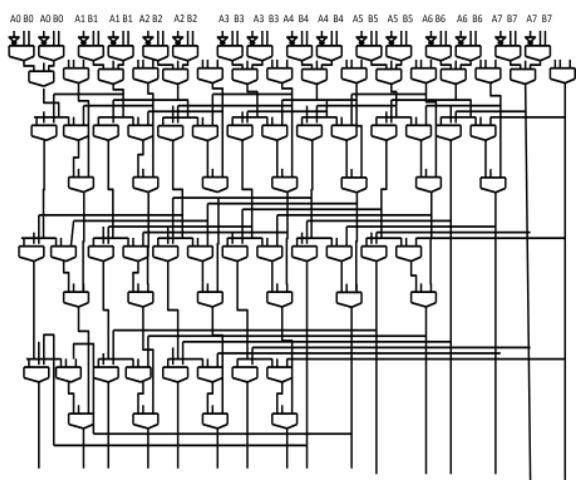

Fig 12: Kogge Stone adder with majority gates

The HMPE structure is shown in Fig 13, in which Parallel prefix adder and Modified excess one unit are important. These two are designed and implemented in QCA technology using majority gates. The XOR gates and AND gates are designed in individually with two input majority gates. Two input majority gate is same as 3 input majority gate by taking one input as ' 1 ', or ' 0 ' based on the required operation.

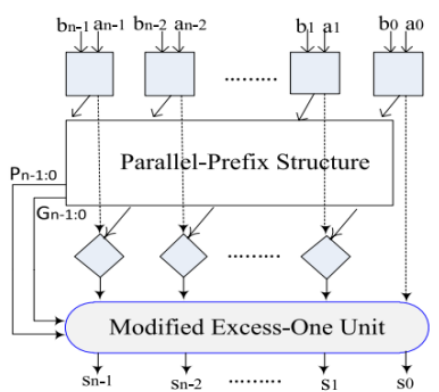

Fig 13: HMPE structure

\section{SIMULATION RESULTS OF HMPE AND HRPX STRUCTURES USING KS AND BK ADDERS}

For HMPE adders, any prefix adder is preffered. It is implemented using KS and BK adders. The input operands ' $a$ ' and ' $b$ ' are of 8-bits and the output result is shown in sum operand. Here the outcome of addition is incremented by one as it contains excess one unit for single zero representation. The RTL Schematic and simulation result of implementation of HMPE using KS adder are shown in Fig 14 and 15 respectively. 


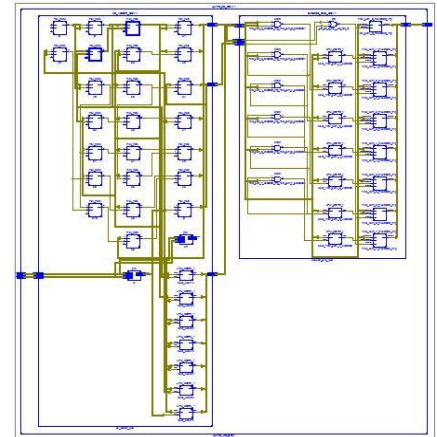

Fig 14: Simulation result of HMPE using KS structure

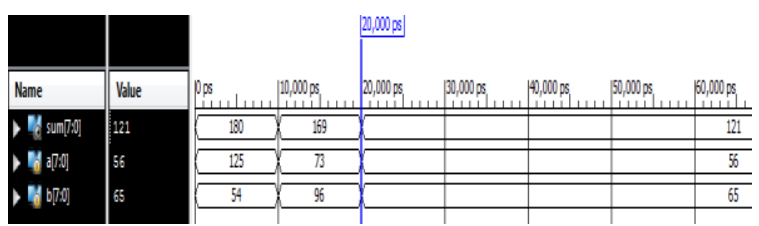

Fig 15: Simulation result of HMPE using KS structure

The RTL Schematic and simulation result of implementation of HMPE using KS adder are shown in Fig 16 and 17 respectively.

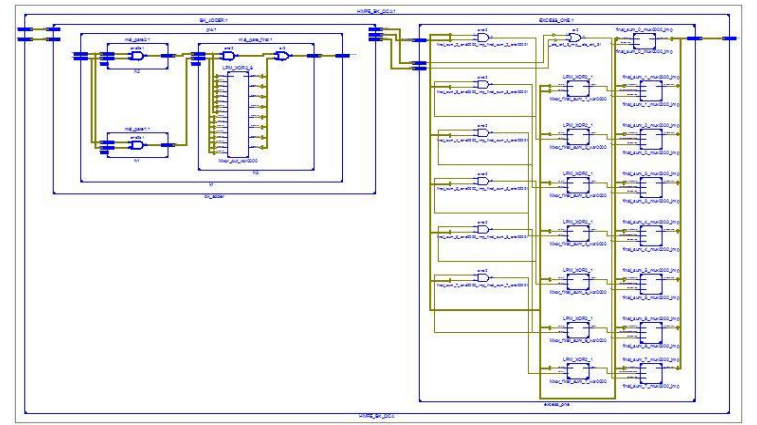

Fig 16: Simulation result of HMPE using BK structure

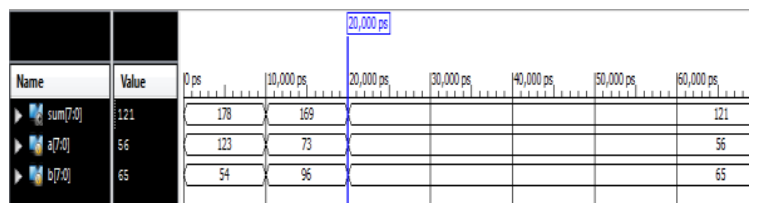

Fig 17: Simulation result of HMPE using BK structure

For HRPX adders, 'a' and 'b' are taken of 8-bit and 18 bit of size respectively and their sum is evaluated at the output sum operand. As it is modulo addition, the size of the input of the input values varies. The RTL Schematic and simulation result of implementation of HRPX using BK adder are shown in Fig 18 and 19 respectively.

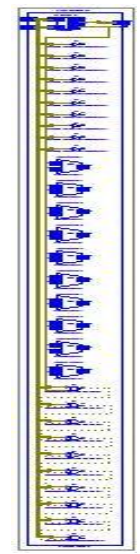

Fig 18: Simulation result of HRPX using BK structure

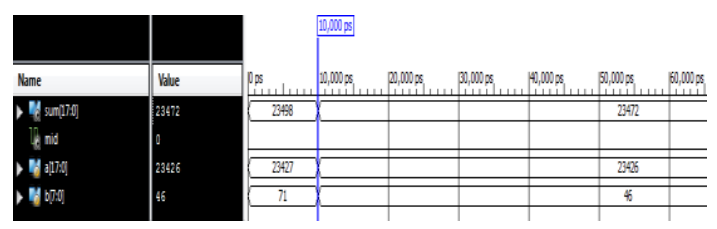

Fig 19: Simulation result of HMPE using BK structure

\section{POWER ANALYSIS USING POWER ANALYZER}

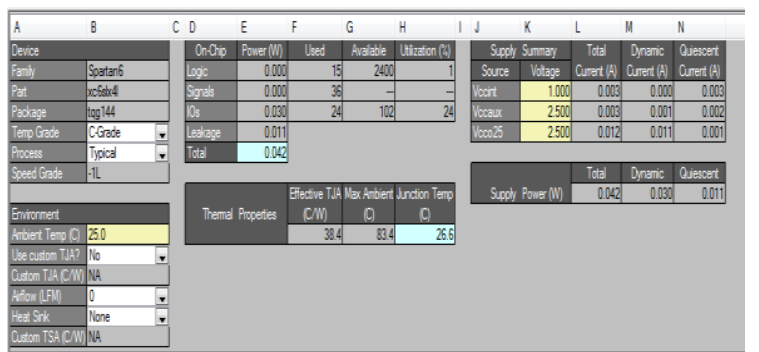

Fig 20: Power analysis using XPower Analyzer.

From the above picture, the dynamic power is shown by Xilinx tool with XPower Analyzer. XPower Analyzer provides the detailed power analysis by using mapping report file generated during the synthesis using Xilinx synthesizer. The dynamic power is $0.030 \mathrm{~W}$ and static power is $0.011 \mathrm{~W}$. The total power is $0.042 \mathrm{~W}$.

Table 1. Comparison Table in Terms of Power and Delay

\begin{tabular}{|c|c|c|c|}
\hline & $\begin{array}{c}\text { HRPX } \\
\text { BK }\end{array}$ & $\begin{array}{c}\text { HMPE } \\
\text { BK }\end{array}$ & HMPE KS \\
\hline Power(W) & 0.059 & 0.042 & 0.014 \\
\hline $\begin{array}{c}\text { Power with } \\
\text { QCA }\end{array}$ & 0.062 & 0.041 & 0.014 \\
\hline Delay(ns) & 16.359 & 14.389 & 14.569 \\
\hline $\begin{array}{c}\text { Delay with } \\
\text { QCA }\end{array}$ & 14.875 & 13.972 & 14.403 \\
\hline
\end{tabular}

From the above table, it is shown that the delay is reduced but the power is increased for HRPX structure. For HPME using BK adder, both the delay and power are reduced and it is shown that there is slight difference in delay and power when this structure implemented with KS. 


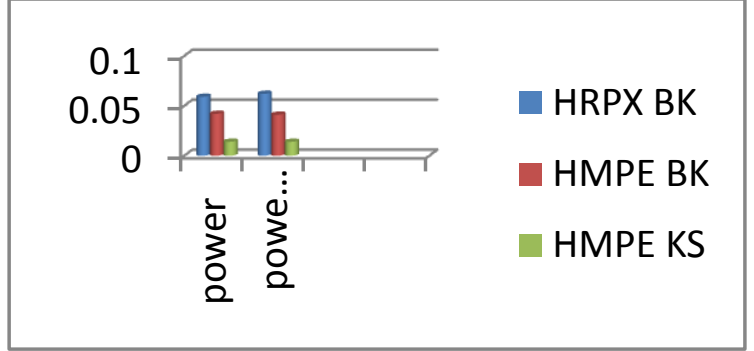

Fig 21: Performance Analysis for power

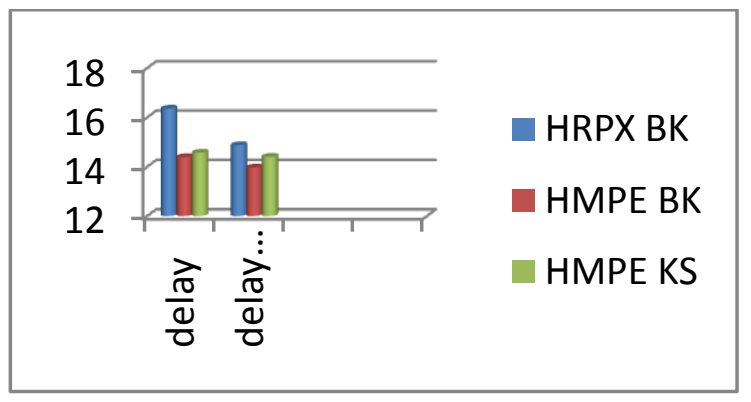

Fig 22: Performance analysis for Delay

\section{CONCLUSION}

The hybrid parallel prefix adders used in reverse converter are designed, which performs the addition for moduli sets. Parallel prefix adders are employed with the hybrid adders to achieve high speed computation and low power consumption. The entire work is done in Xilinx 13.2 ISE simulator. All these structures are simulated and verified by the application of QCA technology which resulted in higher speed when compared with the implementation of adders without QCA technology.

\section{REFERENCES}

[1] Y. Wang , X. Song, M. Aboulhamid, and H. Shen, "Adder based residue to binary numbers converters for $(2 \mathrm{n}-1,2 \mathrm{n}, 2 \mathrm{n}+1)$, "IEEE Trans. SignalProcess., vol.50, no.7, pp.1772-1779, Jul. 2002.

[2] R. A. Patel, M. Benaissa, and S. Boussakta, "Fast parallel-prefix architectures for modulo $2 \mathrm{n}-1$ addition with a single representation of zero", IEEE Trans. Comput., vol. 56, no.11, pp.1484-1492, Nov. 2007.

[3] A. Omondi and B. Premkumar, Residue Number Systems: Theory and Implementations. London, U.K.: Imperial College Press, 2007.

[4] B. Parhami, Computer Arithmetic: Algorithms and Hardware Designs, 2nd ed., New York, NY, USA: Oxford Univ. Press, 2010.

[5] K. Navi, A. S. Molahosseini, and M. Esmaeildoust, "How to teach residue number system to computer scientists and engineers," IEEE Trans. Educ., vol.54, no.1, pp.156-163, Feb.2011.

[6] B. Ramkumar and H. M. Kittur, "Low power and area efficient carry select adder," IEEE Trans. Very Large ScaleIntegr.(VLSI)Syst.,vol.20,no.2,pp.371375,Feb.2012.

[7] L. Sousa and S. Antao, "MRC-based RNS reverse converters for the four-moduli sets $\{2 n+1,2 n-1,2 n$, $22 \mathrm{n}+1-1\}$ and $\{2 \mathrm{n}+1,2 \mathrm{n}-1,22 \mathrm{n}, 22 \mathrm{n}+1-1\}$, IEEE Trans. Circuits Syst. II, vol. 59, no. 4, pp. 244-248, Apr. 2012.

[8] A. S. Molahosseini, S. Sorouri, and A. A. E. Zarandi, "Research challenges in next-generation residue number system architectures," in Proc. IEEE Int. Conf. Comput.Sci.Educ., Jul.2012, pp.1658-1661.

[9] S. Antão and L. Sousa, "The CRNS framework and its application to programmable and reconfigurable cryptography," ACM Trans.Archit.CodeOptim., vol.9, no.4, pp.33, Jan.2013.

[10] J. Chen and J. Hu, "Energy-efficient digital signal processing via voltageover scaling-based residue number system," IEEE Trans. Very Large Scale Integr. (VLSI) Syst., vol.21, no.7, pp.1322-1332,Jul.2013.

[11] C. H. Vun, A. B. Premkumar, and W. Zhang, "A new RNS based DA approach for inner product computation," IEEE Trans. Circuits Syst. I, Reg. Papers, vol. 60, no. 8, pp. 2139-2152, Aug.2013

\section{AUTHOR PROFILE}

Ms. N.Chandini is currently pursuing her M.tech in VLSI system design in Electronics and Communication Engineering Department, AITAM, Tekkali. Her areas of interest are low power VLSI system design and digital filter optimization. The author may be reached at chandinichandu96@gmail.com.

Mr.B.ChinnaRao is presently working as an Associate Professor in Electronics and Communication Engineering Department, AITAM, Tekkali.He is pursuing his Ph.D from JNTUH in the department of Electronics and Communication Engineering. He has 16 years experience in teaching and research. He published more than 25 research papers in National/International Journals and Conferences. He is a life member of ISTE and IE and a member of IEEE.

Mrs.A.JayaLaxmi is currently work ing as an Assistant Professor in Electronics and Communication Engineering Department, AITAM, Tekkali. She completed her M.tech from JNTU, Kakinada in the Department of Electronics and Communication. She has 10 years experience in the teaching and research. She published 5 national/international Journals and Conferences. 